\title{
ARTICLE
}

Received 23 Apr 2010 | Accepted 28 Jul 2010 | Published 24 Aug 2010

DOI: $10.1038 /$ ncomms1056

\section{Intrinsic magnetism at silicon surfaces}

\author{
Steven C. Erwin' \& F.J. Himpsel ${ }^{2}$
}

It has been a long-standing goal to create magnetism in a non-magnetic material by manipulating its structure at the nanoscale. Many structural defects have unpaired spins; an ordered arrangement of these can create a magnetically ordered state. In this article we predict theoretically that stepped silicon surfaces stabilized by adsorbed gold achieve this state by selfassembly, creating chains of polarized electron spins with atomically precise structural order. The spins are localized at silicon step edges having the form of graphitic ribbons. The predicted magnetic state is supported by recent experimental observations, such as the coexistence of double- and triple-period distortions and the absence of edge states in photoemission. Ordered arrays of surface spins can be accessed by probes with single-spin sensitivity, such as spinpolarized scanning tunnelling microscopy. The integration of structural and magnetic order is crucial for technologies involving spin-based computation and storage at the atomic level. 
ndividual atoms with an odd number of electrons have a magnetic moment from the spin of the unpaired electron. Materials consisting of elements with an even number of electrons-such as carbon, silicon and oxygen-can also reveal unpaired electrons when covalent bonds are broken. In defective or disordered group IV solids, the single electrons that occupy defect levels can be detected in magnetic resonance experiments ${ }^{1}$, but the typically low concentrations of these defects normally preclude a magnetically ordered state. Recent intriguing reports of ferromagnetism in insulating oxides have led to the idea of ' $d^{0}$ magnetism', in which magnetic order is argued to arise purely from spin-polarized native defects such as oxygen or cation vacancies ${ }^{2}$. These phenomena remain controversial and their theoretical understanding is incomplete $^{3,4}$. Nevertheless, the pursuit of ferromagnetic materials without transition metals or rare earths is part of a broad effort to identify and develop new systems in which spin-polarized electrons can be used to store and process information.

Bonds are also broken at the surfaces and interfaces of solids, but covalent materials usually reconstruct to eliminate dangling bonds or doubly occupy them with electrons of opposite spin. Surfaces nonetheless provide natural templates for creating ordered arrays of spins that can be individually addressed by local probes. For example, step edges on vicinal $\mathrm{Si}(111)$ can be atomically smooth for distances as great as 20,000 lattice sites ${ }^{5}$. These step edges have already been proposed as templates for creating long chains of nuclear spins, consisting of ${ }^{29} \mathrm{Si}$ atoms, as the basis for an all-silicon quantum computer ${ }^{6}$.

Here, we describe a very different alternative to fabricating chains of spins on templated surfaces. The concept rests on our prediction that a family of well-known vicinal silicon surfaces are themselves intrinsically magnetic. Thus, these surfaces create-entirely by selfassembly-long chains comprising thousands of electron spins with nearly perfect structural order. Such ordered arrays of spins in silicon systems having no magnetic elements are quite unexpected. Indeed, none of these systems has yet been investigated for magnetism experimentally, but we show below that existing data already support it indirectly.

\section{Results}

Spin polarization at graphitic steps in silicon. Our investigation focuses on $\mathrm{Si}(553)$-Au and $\mathrm{Si}(557)$-Au surfaces, shown in Figure 1.
These are stepped surfaces stabilized by a fraction of a monolayer of $\mathrm{Au}$, and are members of a family of Au-induced vicinal silicon surfaces with orientations close to (111). The Si(553)-Au surface is the most perfect of the Au-induced chain structures on vicinal $\mathrm{Si}(111)$ : its structural perfection is limited only by defects created by adsorption of residual gas. Even these will be frozen out at the temperatures normally used (below $5 \mathrm{~K}$ ) to probe individual spins at surfaces ${ }^{7}$. Crystallographically, $\mathrm{Si}(553)$ and (557) can be considered miscut from the flat $\mathrm{Si}(111)$ surface by angles of $12.3^{\circ}$ and $9.4^{\circ}$ away from and towards, respectively, the (001) orientation. Several other Au-stabilized vicinal (111) surfaces contain structural motifs in common with $\mathrm{Si}(553)-\mathrm{Au}$ and $\mathrm{Si}(557)-\mathrm{Au}^{8}$. These commonalities suggest that magnetism in silicon is likely to be found throughout this family.

The critical structural motif for magnetism in these systems is a nanoscale honeycomb strip of graphitic silicon, just a single hexagon wide, which forms the edge of the step ${ }^{9}$. This honeycomb chain was first observed in reconstructions of flat $\mathrm{Si}(111)$ induced by submonolayer coverages of alkali, alkaline earth, and some rare-earth metals as well as $\mathrm{Ag}$ and $\mathrm{Au}^{10}$. In these flat surfaces, the orbitals of the outer honeycomb atoms form filled bands, either by accepting electrons from the adsorbates or by forming partially covalent bonds. At a step edge, a new possibility arises in which these orbitals are singly occupied, fully spin polarized and magnetically ordered.

The two surfaces studied here have step edges with opposite orientation, as shown in Figure 1. We used density-functional theory (DFT) to determine the equilibrium structure of these surfaces. The results are shown in Figure 1a,b for $\mathrm{Si}(553)$-Au and in Figure 1c for $\mathrm{Si}(557)$-Au. The same structural model for $\mathrm{Si}(553)$-Au was found independently in ref. 11 on the basis of non-spin-polarized DFT calculations. Similarly, the model of $\mathrm{Si}(557)-\mathrm{Au}$ is well established from earlier non-spin-polarized DFT calculations ${ }^{8,12}$ and from $\mathrm{X}$-ray diffraction ${ }^{13}$.

By including spin polarization in our calculations, we find that some of the dangling bonds at the step edges of both surfaces become fully polarized with a spin moment of one Bohr magneton. On $\mathrm{Si}(553)$-Au, every third $\mathrm{Si}$ atom at the step edge is completely spin polarized, whereas all other atoms are negligibly polarized. On $\mathrm{Si}$ (557)-Au (Fig. 1c), every second Si atom at the step is fully polarized, as are all the terrace restatoms. In both systems, the interaction
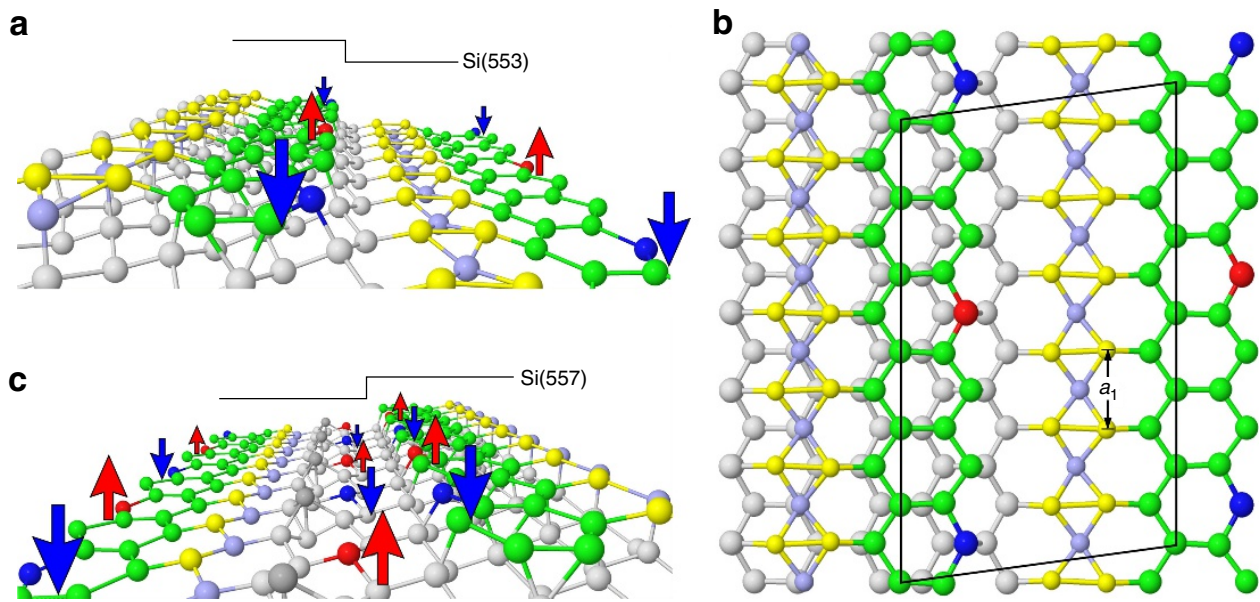

Figure 1 | Ground state structure and lowest energy spin configuration of two magnetic silicon surfaces. (a, b) Si(553)-Au in its antiferromagnetic ground state. Yellow atoms are Au, all others are Si. Each terrace contains a Au double row and a graphitic Si honeycomb chain (green) at the step edge. Every third Si (red, blue) at the step has a spin magnetic moment of one Bohr magneton ( $S=1 / 2$, arrows) from the complete polarization of the electron occupying the dangling-bond orbital. The sign of the polarization alternates along the step. The periodicity along the step is tripled by a small downward displacement of the magnetic atoms. The periodicity along the two Au rows is doubled, with alternating short and long bonds $a_{1}$. The full $1 \times 6$ unit cell is outlined. (c) Si(557)-Au in its antiferromagnetic ground state. The step direction of Si(557) is opposite to that of Si(553) as shown by the schematic outlines. Nevertheless, this surface is also magnetic: every second Si (red, blue) at the step has a magnetic moment of one Bohr magneton, as do all the Si restatoms (blue, red) on the terrace. 
between neighbouring spins along the step is antiferromagnetic. In the spin-polarized antiferromagnetic ground state, the total energy of the system is lower than in the non-spin-polarized state. For $\mathrm{Si}(553)-\mathrm{Au}$, this energy gain is $20 \mathrm{meV}$ per spin (see Supplementary Discussion and Supplementary Fig. S1), suggesting that spin polarization will be important only well below room temperature.

For Si(553)-Au, the spin-polarized ground state solves two outstanding experimental puzzles: (1) scanning tunnelling microscopy (STM) of $\mathrm{Si}(553)$-Au reveals the coexistence of two distortions with different periods at low temperatures. We show that these competing periodicities are not only correctly predicted but, in addition, are inextricably linked with the spin-polarized ground state. Their experimental observation constitutes indirect but compelling evidence supporting the possibility of magnetism in $\mathrm{Si}(553)$-Au. (2) The non-spin-polarized band structure, although largely in agreement with experiment for the Au chains, predicts sharp step-edge states not seen in photoemission ${ }^{11}$. These 'missing states' can now be understood as being washed out by fluctuations in the exchange splitting of the spin-polarized electronic structure.

For $\mathrm{Si}(557)-\mathrm{Au}$, the spin-polarized calculation resolves a different experimental puzzle: the observation of a metal-insulator transition at low temperature. An insulating ground state, with a small gap, is indeed found in the spin-polarized ground state, whereas the non-spin-polarized band structure is metallic. However, the most intense energy bands in photoemission already agree well with the calculated non-spin-polarized bands arising from the Au chains ${ }^{12}$. This agreement remains equally good in the spin-polarized description. In both descriptions, a small band splitting ${ }^{14-17}$ originates from the spin-orbit interaction of the $\mathrm{Au}$ atoms ${ }^{12,18}$. In this 'Rashba effect'-which is unrelated to the magnetism we predict herethe two split bands have a spin polarization of $100 \%$ at any given momentum in reciprocal space, but this vanishes when integrated over all momenta and leaves no net spin polarization in real space.

Predictions for $\mathrm{Si}(553)-\mathrm{Au}$. The $\mathrm{Si}(553)-\mathrm{Au}$ surface was first observed in ref. 19 and its unusual one-dimensional electronic properties have since been extensively studied. Two experimental findings are especially striking: (1) STM images acquired at room temperature show alternating bright and dim rows, each with the periodicity of the Si surface lattice constant $a_{0}{ }^{20-23}$. At temperatures below $50 \mathrm{~K}$ these rows separately develop higher-order periodicity, with a tripled period $\left(3 a_{0}\right)$ for the bright rows and a doubled period $\left(2 a_{0}\right)$ for the dim rows. These effects have been attributed to periodic lattice distortions ${ }^{21}$ and to charge-density waves ${ }^{22}$, but their true microscopic origin has remained elusive. (2) Angle-resolved photoemission spectra (ARPES) obtained at 160-220 K reveal three metallic bands, all with their minima at the zone boundary (in the direction parallel to the chains) $\mathrm{ZB}_{\|}$of the $1 \times 1$ Brillouin zone ${ }^{19}$. The two lower bands are slightly split by a small momentum splitting $\delta k_{\mathrm{F}}=0.05 \AA^{-1}$ (ref. 18), and cross the Fermi level about halfway to the zone centre. A detailed analysis of this crossing shows that the splitting is due to the spin-orbit interaction ${ }^{18}$. The upper band crosses the Fermi level about one-fourth of the way to the zone centre without a detectable splitting.

All these observations are correctly predicted by the proposed model of Si(553)-Au. Figure 2 shows the theoretically simulated STM image for the model in Figure 1a in its antiferromagnetic ground state. The bright row arises from $\mathrm{Si}$ atoms at the step edge and indeed has tripled periodicity $3 a_{0}$. The highest peaks within this row are from the spin-polarized atoms. This appears counterintuitive, as these atoms are actually $0.3 \AA$ below the height of their non-polarized neighbours. But each spin-polarized atom has one unoccupied orbital just above the Fermi level, whereas the nonpolarized atoms have only occupied orbitals. For tunnelling into empty states, the topographic peaks are therefore indeed higher for spin-polarized atoms.
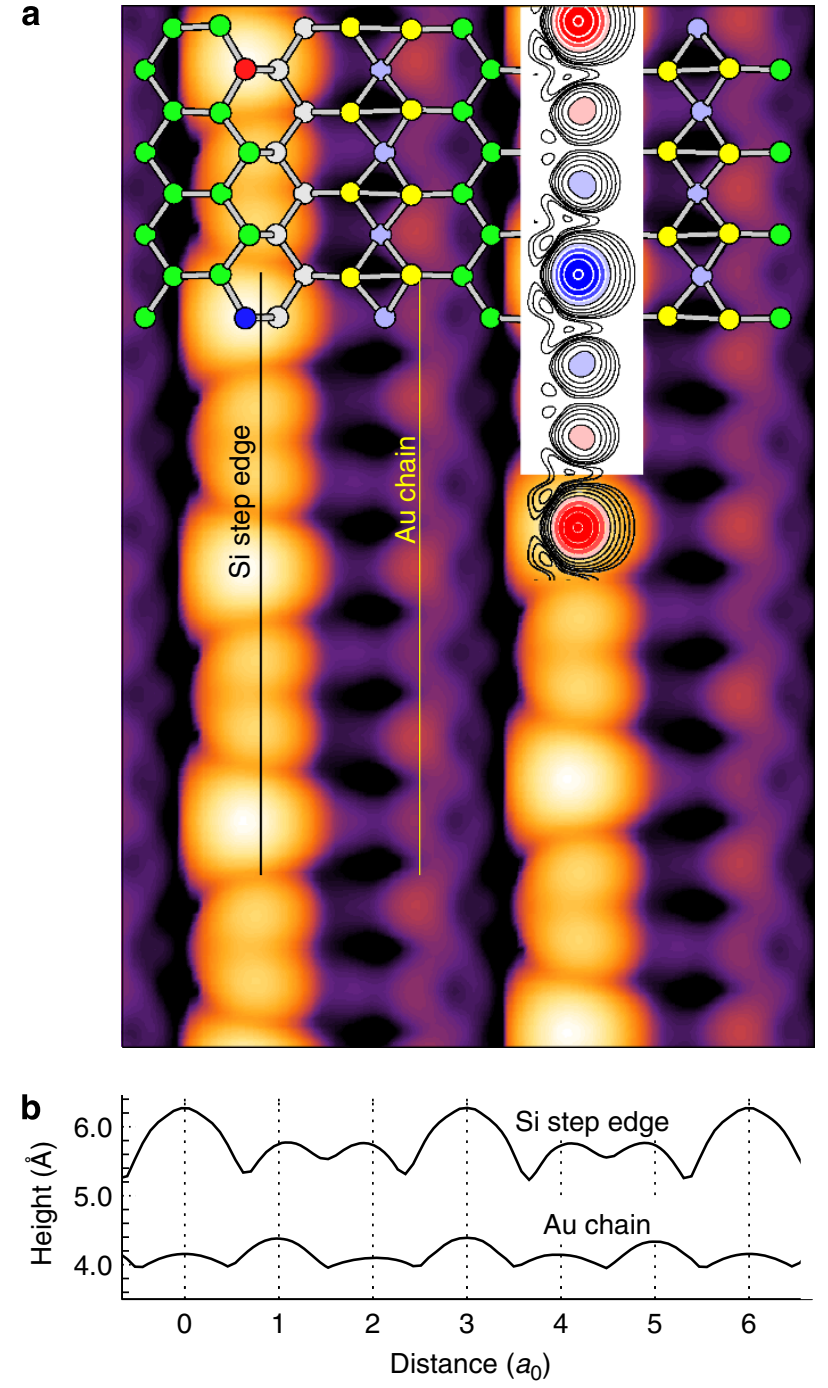

Figure 2 | Scanning tunnelling microscopy of Si(553)-Au. (a) Theoretical STM topography showing the tip height at constant current for tunnelling into empty surface states at bias voltage $+0.5 \mathrm{~V}$. Inset: electron spin density $1 \AA$ above the step edge. Red and blue contours represent positive and negative polarization, indicating antiferromagnetic alignment of the red and blue Si atoms the step edge. Contours are separated by a factor of two. (b) Line scans at the locations marked in $\mathbf{a}$, showing coexisting $3 a_{0}$ and $2 a_{0}$ periodicities along the Si step edge and Au chain, respectively.

The dim second row visible in Figure 2a arises from the righthand leg of the ladder formed by two rows of $\mathrm{Au}$ atoms. Its doubled periodicity $2 a_{0}$ is due to the dimerization created when the rungs of this ladder rotate with alternating signs along a row. A similar dimerization occurs in a closely related system, $\mathrm{Si}(111)-(5 \times 2)-\mathrm{Au}$, where it is driven by electron doping from $\mathrm{Si}$ adatoms and the consequent formation of a surface band gap ${ }^{24}$. For $\mathrm{Si}(111)-(5 \times 2)-\mathrm{Au}$, the dimerization parameter $d=\left(a_{1}-a_{0}\right) / a_{0}$, where $a_{0}$ is the surface lattice constant and $a_{1}$ is defined in Figure $1 \mathrm{~b}$, has the value $d=0.14$, much larger than the value $d=0.04$ for $\mathrm{Si}(553)$-Au. But even this small dimerization is sufficient to produce a doubled periodicity that is well resolved in the theoretical STM image.

The spin polarization of the electron density, shown in the inset of Figure $2 \mathrm{a}$, is strongly localized at every third Si step-edge atom. Integrating this spin density shows that the red and blue step-edge atoms each have a spin moment of one Bohr magneton, equivalent to a fully polarized electron with spin $S=1 / 2$. The direction of the spin alter- 
nates along the step in this antiferromagnetic ground state. Moments on other atoms along the step are smaller by an order of magnitude.

The connection between magnetism and the periodicity of the rows is simple and direct. If the spin polarization is constrained to be zero, the DFT equilibrium geometry of the model changes: both the tripled periodicity along the Si rows and the doubled periodicity along the $\mathrm{Au}$ rows are completely eliminated. In this sense, the experimental observation of triple and double periodicities constitutes strong evidence for a spin-polarized ground state.

Magnetism also provides the key to understanding a long-standing puzzle presented by ARPES data for Si(553)-Au. The orbitals at the step edges of a vicinal surface would normally give rise to sharp electronic states near the Fermi level, as is indeed the case for previously proposed models of $\mathrm{Si}(553)-\mathrm{Au}^{25,26}$. But these are not observed in the ARPES data. We demonstrate below that spin polarization at the step edge provides the resolution to this puzzle.

Figure 3a shows the theoretical bands for Si(553)-Au unfolded into the extended Brillouin zone, where they can be easily compared with those obtained from ARPES spectra. As the theoretical ground state is antiferromagnetic, one need only analyse the bands from a single spin channel. The symbols and curves identify, using the colours from Figure 1, the states having the largest projection onto particular atoms at the surface. States arising from the magnetic atoms experience an exchange splitting of about $0.5 \mathrm{eV}$. As a result, for spin-up states, the red step-edge band is singly occupied and the blue step-edge band is empty. For spin-down states, this situation is reversed, as seen in the density of states (DOS; Figure 3b). A more accurate treatment of electron correlation (beyond DFT) could shift these narrow bands somewhat, but the large exchange splitting suggests that qualitative changes are unlikely.

Although these magnetic step-edge states are responsible for the $1 \times 6$ structural and magnetic periodicity of $\mathrm{Si}(553)-\mathrm{Au}$, the remaining features of the band structure have a simple $1 \times 1$ non-magnetic character. This is evident from comparing the $1 \times 6$ bands with those in Figure $3 \mathrm{~d}$ for a simpler $1 \times 1$ structure identical to our model but constrained to unit periodicity $a_{0}$ and thus having no spin polarization. In this $1 \times 1$ structure, there is just a single type of step atom and therefore only one corresponding band (green). The remnant of this non-magnetic band is also visible in the full $1 \times 6$ bands, although there its spectral weight at a given momentum is spread out by partial hybridization with its magnetic counterparts.

a

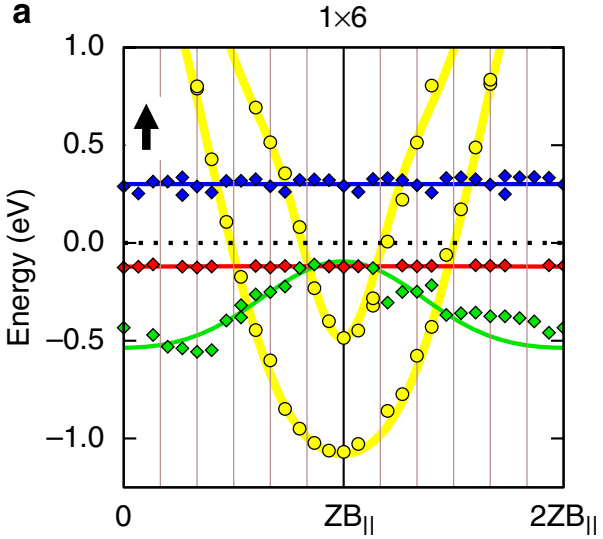

b $1 \times 6$

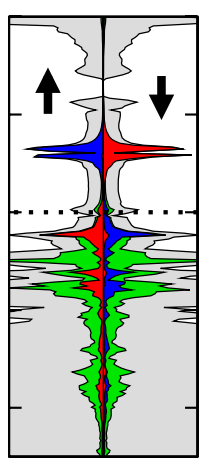

DOS
Both the $1 \times 6$ and $1 \times 1$ structures give rise to several metallic bands (yellow) with their minima at the zone boundary $\mathrm{ZB}_{\|}$. These are the bands detected in ARPES (Fig. $3 \mathrm{~d}$, inset). In the $1 \times 6$ case, there are four bands in the two spin channels, but the spin-up and spin-down bands are nearly degenerate. In the non-magnetic $1 \times 1$ case, there are also four bands, but here the degeneracy is broken by the spin-orbit interaction ${ }^{27}$, which, for simplicity, was not included in the $1 \times 6$ calculation. The resulting momentum splitting $\delta k_{\mathrm{F}}=0.04 \AA^{-1}$ of the lower band is in excellent agreement with the experimental splitting. The splitting of the upper band is less than half this value and consequently is not resolved in the ARPES data. The predicted Fermi level crossings of these bands are in qualitative agreement with the measured values: the lower doublet crosses the Fermi level halfway to the zone centre, and the upper doublet about one-fifth of the way.

One feature of the non-magnetic $1 \times 1$ band structure is not observed in the ARPES data: the green step-edge band that just crosses the Fermi level in Figure 3d. Magnetism provides a simple explanation for the apparent absence of this band. Above a temperature scale set by the energy gained from spin polarization, thermal fluctuations between polarized and unpolarized electron states will broaden the step-edge bands. The magnitude of the broadening is roughly the exchange splitting, $0.5 \mathrm{eV}$. The resulting reduction in intensity makes the step-edge bands essentially invisible compared with the sharp Au bands, which are not affected by the spin polarization at the step edge. In this sense the experimental absence of a sharp step-edge band constitutes additional evidence for a spinpolarized ground state.

Predictions for Si(557)-Au. We now briefly summarize our predictions for the magnetic properties of $\mathrm{Si}(557)$ - Au. The main features are similar to those of $\mathrm{Si}(553)$-Au, with several interesting differences.

(1) Crystallographically, the steps on $\mathrm{Si}(557)$ are oriented in an opposite manner to those on $\mathrm{Si}(553)$. On clean, unreconstructed silicon, this difference would lead to two dangling bonds at each stepedge atom, in contrast to the single dangling bond at each step-edge atom of $\mathrm{Si}(553)$. But the graphitic reconstruction of the steps on $\mathrm{Si}(557)-\mathrm{Au}$ leads, just as for $\mathrm{Si}(553)-\mathrm{Au}$, to a single dangling bond per step-edge atom. We find that every second of these dangling bonds is fully spin polarized. This $2 a_{0}$ periodicity in spin polarization tracks the up-down buckling of the step-edge atoms previously

Figure 3 | Electronic band structure and density of states (DOS) for Si(553)-Au. (a, b) Bands and DOS for the ground-state antiferromagnetic structure of Figure 1a. The full $1 \times 6$ bands are shown unfolded and parallel to the chain direction ( $Z B_{\|}$is the boundary of the $1 \times 1$ Brillouin zone) along the line halfway to the orthogonal zone boundary $Z B_{\perp}$. Red and blue diamonds are spin-polarized states from red and blue Si step-edge atoms in Figure 1a. Only spin-up states are shown here-antiferromagnetic order implies the spin-down states are equivalent but with red and blue reversed, as seen in the DOS. Green diamonds are non-polarized states from green Si step-edge atoms. Yellow circles are bonding (lower) and anti-bonding (upper) states from the Au chains. Curves show schematic energy bands without hybridization. Projected bulk states are not shown, for clarity. (c, d) Bands and DOS for the same model when it is constrained to $1 \times 1$ periodicity, for which the ground state is not spin-polarized. Spin-orbit coupling, included in the bands but not in the DOS, splits each of the two Au chain states but not the Si step-edge state. Inset: experimental angle-resolved photoemission spectra from Crain et al. ${ }^{19}$ 
found in non-spin-polarized calculations ${ }^{12,28}$. Only the 'down' atoms are polarized.

(2) The terraces on $\mathrm{Si}(557)$ - $\mathrm{Au}$ are wider than those on $\mathrm{Si}(553)$ $\mathrm{Au}$ and contain a row of 'restatoms' not found on $\mathrm{Si}(553)-\mathrm{Au}$. We find that the dangling bond of each restatom is singly occupied and fully spin polarized. Because the restatoms are separated by $2 a_{0}$ along the step direction, the number of spin-polarized step-edge atoms and restatoms is the same.

(3) The dominant interaction between neighbouring spins along the step edge is antiferromagnetic; the same is true for the spins along the row of restatoms. Adjacent rows of spins are also preferentially aligned antiparallel. In this antiferromagnetic ground state, the energy of the system is reduced by $45 \mathrm{meV}$ per spin relative to the non-spin-polarized state.

(4) In non-spin-polarized calculations, the restatom orbital is doubly occupied and the orbital of the down step-edge atom is nearly empty $y^{12,28}$. In the spin-polarized ground state, this changes: both the restatom and down step-edge atoms are singly occupied (and polarized). This charge transfer reduces the magnitude of the step-edge buckling and also reduces the height of the restatoms.

(5) There is considerable experimental controversy regarding the possibility that $\mathrm{Si}(557)$-Au undergoes a metal-insulator transition at low temperature ${ }^{17,29-31}$. The origin of an insulating state at low temperature is not clear. The theoretical non-spin-polarized band structure is metallic. It has been proposed that thermal fluctuations at the step edge could contribute to a metal-insulator transition ${ }^{12,28}$. An alternative explanation is found in the spin-polarized band structure, which is insulating with a small gap of $75 \mathrm{meV}$ (which persists when spin-orbit coupling is included).

(6) The first theoretical studies of Si(557)-Au included spin-orbit coupling but not spin polarization ${ }^{12}$. The resulting prediction of momentum-split Au bands was in good agreement with experiments. These studies also predicted Si step-edge and restatom bands not seen in ARPES ${ }^{8,15,17}$. In Riikonen et al..$^{28}$ it was demonstrated theoretically that thermal fluctuations cause these bands to oscillate with an amplitude of approximately $0.2 \mathrm{eV}$ and can thus blur these nominally sharp states. By including spin polarization in the calculation, another scenario emerges. The exchange splitting in $\mathrm{Si}(557)-\mathrm{Au}$ pushes the two occupied spin bands down to $0.4-0.5 \mathrm{eV}$ below the Fermi level, and the two unoccupied spin bands up to $0.1-0.2 \mathrm{eV}$ above the Fermi level. This exchange splitting, approximately $0.5 \mathrm{eV}$, is similar to that we found for $\mathrm{Si}(553)$-Au. Thermal fluctuations between polarized and unpolarized states will similarly broaden these step-edge and restatom bands, rendering them invisible to ARPES while leaving the momentum-split Au bands unaffected. The scale of the fluctuations in both scenarios is comparable, and thus it is likely that both mechanisms contribute to blurring the energy bands.

(7) In contrast to our findings for $\mathrm{Si}(553)$-Au, we do not predict any higher-order periodicity to mark the onset of magnetism at low temperature: the periodicity of $\mathrm{Si}(557)$-Au remains $1 \times 2$ in both the non-spin-polarized and spin-polarized states.

\section{Discussion}

We anticipate that spin-polarized states in silicon are not limited to $\mathrm{Si}(553)-\mathrm{Au}$ and $\mathrm{Si}(557)-\mathrm{Au}$, because the key structural element-a graphitic step edge-is known to exist on other stepped silicon surfaces as well ${ }^{8}$. Direct experimental tests of our predictions using, for example, spin-polarized STM ${ }^{32,33}$ or spin-polarized photoemission ${ }^{34}$ will be an important next step, and will provide important information about the nature of the magnetic ordering.

To set the stage for such tests, it is helpful to estimate the magnitude of the spin interactions within a simple nearest-neighbour Heisenberg Hamiltonian. From DFT total-energy calculations of different spin configurations on $\mathrm{Si}(553)$ - $\mathrm{Au}$, we find antiferromagnetic coupling $\left(J_{\|}=15 \mathrm{meV}\right)$ along the steps and weaker, ferromagnetic coupling $\left(J_{\perp}=-0.3 \mathrm{meV}\right)$ across the steps (these values may be different in methods that treat electron correlation explicitly; see the discussion in Volnianska et al. ${ }^{35}$ ). Intriguingly, we find that the magnitude, and even the sign, of these couplings can be changed by doping electrons or holes into the Si(553)-Au surface states (see Supplementary Methods and Supplementary Fig. S2). It is well established that surface states on the closely related $\mathrm{Si}(111)$-Au surface can be electron-doped by adsorbates (for example, silicon adatoms) on the surface, and that by varying this adsorbate population one can perform band-structure engineering with extraordinary preci$\operatorname{sion}^{36}$. The possibility of tuning surface magnetism using adsorbate dopants, or surface chemistry more generally, suggests a fascinating new research direction.

Linear chains of spin-polarized atoms provide atomically perfect templates for the ultimate memory and logic, in which a single spin represents a bit. Here, the spins are locked into a self-assembled rigid lattice on a substrate that is compatible with silicon technology. One potential application is the spin shift register recently proposed theoretically by Mahan ${ }^{37}$. This device requires a one-dimensional chain of identical atoms, each with spin $S=1 / 2$. When an additional electron is conducted along the chain, each spin state is shifted by one atom-the spin analogue of a standard shift register memory device. The arrangement of spins is arbitrary and there is no requirement of long-range order. However, two other important criteria must be satisfied: in the ground state each atom must have one electron, and the atoms must have correlated electronic states. The first criterion is indeed satisfied by the spin-polarized atoms on $\mathrm{Si}(553)-\mathrm{Au}$ and $\mathrm{Si}(557)-\mathrm{Au}$. In previous research we showed that the electronic states of singly occupied silicon dangling bonds are indeed strongly correlated ${ }^{38}$.

Another potential application is the storage of information in single magnetic atoms $\mathrm{s}^{39}$. Towards this end, Hirjibehedin et al. ${ }^{40}$ recently used spin excitation spectroscopy to demonstrate that the orientation of an individual spin can be measured with an STM. For information storage, the stability of this orientation, for example from coupling of the spin to the substrate, is critical. This coupling is most likely to be substantial when the substrate is anisotropic at the atomic scale. In the experiments by Hirjibehedin, this anisotropy was engineered by creating $\mathrm{Cu}_{2} \mathrm{~N}$ islands that break the four-fold symmetry of the $\mathrm{Cu}(001)$ substrate. In $\mathrm{Si}(553)$-Au and related vicinal silicon surfaces, this anisotropy arises naturally at the step edge. The investigation of spin-lattice coupling in these magnetic silicon surfaces is an important next step towards the ultimate goal of spintronics with single spins.

\section{Methods}

Density-functional theory calculations. First-principles total-energy calculations were used to determine equilibrium geometries and relative energies of the models in Figure 1 and their variants. The calculations were performed in a slab geometry with six or more layers of Si along with the reconstructed top surface layer and a vacuum region of at least $10 \AA$. All atomic positions were relaxed, except the bottom $\mathrm{Si}$ layer and its passivating hydrogen layer, until the largest force component on every atom was below $0.02 \mathrm{eV} / \AA$. Total energies and forces were calculated within the generalized-gradient approximation of Perdew, Burke and Ernzerhof ${ }^{4}$ to DFT using projector-augmented wave potentials, as implemented in VASP ${ }^{42,43}$ The plane-wave cutoff for all calculations was $250 \mathrm{eV}$.

Brillouin-zone sampling. The sampling of the surface Brillouin zone was chosen according to the size of the surface unit cell and the relevant precision requirements. For the $1 \times 6$ reconstruction of $\mathrm{Si}(553)$-Au shown in Figure 1a,b, we calculated the equilibrium geometries and total energies of non-magnetic, ferromagnetic and antiferromagnetic configurations using $6 \times 6$ zone sampling, with convergence checks using $10 \times 10$ sampling. For the $1 \times 2$ reconstruction of $\mathrm{Si}(557)$-Au shown in Figure 1c, we calculated the equilibrium geometries and total energies of nonmagnetic, ferromagnetic and antiferromagnetic configurations using $2 \times 8$ zone sampling, with convergence checks using $2 \times 16$ sampling.

Exchange coupling. Exchange coupling constants $J_{\|}$and $J_{\perp}$ for $\mathrm{Si}(553)$-Au were extracted from DFT total energies. Supercells containing two spins on each of two adjacent steps were constructed with the four possible unique spin configurations. 
The DFT total energies were fit to a nearest-neighbour classical Heisenberg Hamiltonian. The numerical reliability of the coupling constants was analysed with respect to Brillouin-zone sampling, plane-wave cutoff and relativistic treatment, and the dependence of the coupling constants on charge doping of the surface states was investigated (see Supplementary Methods and Supplementary Tables).

Scanning-tunnelling microscopy imagery. The simulated STM image in Figure 2 was calculated using the method of Tersoff and Hamann ${ }^{44}$. For this empty-state image, we integrated the local density of states (LDOS) over an energy window of unoccupied states up to $0.5 \mathrm{eV}$ above the Fermi level. The simulated STM topography under constant-current conditions was obtained by plotting the height at which the integrated LDOS is constant.

\section{References}

1. Elkin, E. L. \& Watkins, G. D. Defects in irradiated silicon: electron paramagnetic resonance and electron-nuclear double resonance of arsenic- and antimony-vacancy pairs. Phys. Rev. 174, 881-897 (1968).

2. Venkatesan, M., Fitzgerald, C. B. \& Coey, J. M. D. Unexpected magnetism in a dielectric oxide. Nature 430, 630 (2004).

3. Chambers, S. A. Ferromagnetism in doped thin-film oxide and nitride semiconductors and dielectrics. Surf. Sci. Reports 61, 345-381 (2006).

4. Osorio-Guillen, J., Lany, S., Barabash, S. V. \& Zunger, A. Magnetism without magnetic ions: percolation, exchange, and formation energies of magnetismpromoting intrinsic defects in CaO. Phys. Rev. Lett. 96, 107203 (2006).

5. Viernow, J. et al. Regular step arrays on silicon. Appl. Phys. Lett. 72, 948-950 (1998).

6. Ladd, T. D. et al. All-silicon quantum computer. Phys. Rev. Lett. 89, 017901 (2002).

7. Yayon, Y., Brar, V. W., Senapati, L., Erwin, S. C. \& Crommie, M. F. Observing spin polarization of individual magnetic adatoms. Phys. Rev. Lett. 99, 067202 (2007).

8. Crain, J. N. et al. Chains of gold atoms with tailored electronic states. Phys. Rev B 69, 125401 (2004).

9. Erwin, S. C. \& Weitering, H. H. Theory of the 'honeycomb chain-channel reconstruction' of M/Si(111)-(3×1). Phys. Rev. Lett. 81, 2296-2299 (1998).

10. Barke, I. et al. Low-dimensional electron gas at semiconductor surfaces. Solid State Comm. 142, 617-626 (2007).

11. Krawiec, M. Structural model of the Au-induced Si(553) surface: double Au rows. Phys. Rev. B 81, 115436 (2010).

12. Sánchez-Portal, D., Riikonen, S. \& Martin, R. M. Role of spin-orbit splitting and dynamical fluctuations in the Si(557)-Au surface. Phys. Rev. Lett. 93 146803 (2004)

13. Robinson, I. K., Bennett, P. A. \& Himpsel, F. J. Structure of quantum wires in Au/Si(557). Phys. Rev. Lett. 88, 096104 (2002).

14. Segovia, P., Purdie, D., Hengsberger, M. \& Baer, Y. Observation of spin and charge collective modes in one-dimensional metallic chains. Nature 402, 504-507 (1999).

15. Losio, R. et al. Band splitting for $\mathrm{Si}(557)$-Au: is it spin-charge separation? Phys. Rev. Lett. 86, 4632-4635 (2001).

16. Altmann, K. N. et al. Electronic structure of atomic chains on vicinal Si(111)Au. Phys. Rev. B 64, 035406 (2001)

17. Ahn, J., Yeom, H., Yoon, H. \& Lyo, I.-W. Metal-insulator transition in Au atomic chains on Si with two proximal bands. Phys. Rev. Lett. 91, 196403 (2003)

18. Barke, I., Zheng, F., Rugheimer, T. K. \& Himpsel, F. J. Experimental evidence for spin-split bands in a one-dimensional chain structure. Phys. Rev. Lett. 97, 226405 (2006).

19. Crain, J. N. et al. Fractional band filling in an atomic chain structure. Phys. Rev. Lett. 90, 176805 (2003).

20. Crain, J. N. \& Pierce, D. T. End states in one-dimensional atom chains. Science 307, 703-706 (2005).

21. Ahn, J. R., Kang, P. G., Ryang, K. D. \& Yeom, H. W. Coexistence of two different Peierls distortions within an atomic scale wire: Si(553)-Au. Phys. Rev. Lett. 95, 196402 (2005)

22. Snijders, P. C., Rogge, S. \& Weitering, H. H. Competing periodicities in fractionally filled one-dimensional bands. Phys. Rev. Lett. 96, 076801 (2006)
23. Crain, J. N., Stiles, M. D., Stroscio, J. A. \& Pierce, D. T. Electronic effects in the length distribution of atom chains. Phys. Rev. Lett. 96, 156801 (2006).

24. Erwin, S. C., Barke, I. \& Himpsel, F. J. Structure and energetics of Si(111) (5×2)-Au. Phys. Rev. B 80, 155409 (2009).

25. Riikonen, S. \& Sánchez-Portal, D. Ab-initio study of the double-row model of the Si(553)-Au reconstruction. Surf. Sci. 600, 1201-1206 (2006).

26. Riikonen, S. \& Sánchez-Portal, D. Systematic investigation of the structure of the Si(553)-Au surface from first principles. Phys. Rev. B 77, 165418 (2008).

27. Koelling, D. D. \& Harmon, B. N. Technique for relativistic spin-polarized calculations. J. Physics C Solid State Phys. 10, 3107-3114 (1977).

28. Riikonen, S. \& Sánchez-Portal, D. Interplay between electronic and atomic structures in the $\mathrm{Si}(557)$-Au reconstruction from first principles. Phys. Rev. B 76, 035410 (2007).

29. Yeom, H. W. et al. Real-space investigation of the metal-insulator transition of Si(557)-Au. Phys. Rev. B 72, 035323 (2005).

30. Kim, H. S. et al. Evidence of metallic nature of the surface bands of $\mathrm{Au} / \mathrm{Si}(557)$. Phys. Rev. B 80, 033412 (2009).

31. Han, J. H. et al. Direct evidence of the step-edge buckling at the $\mathrm{Au} / \mathrm{Si}(557)-1 \times 2$ surface. Phys. Rev. B 80, 241401 (2009).

32. Bode, M. Spin-polarized scanning tunnelling microscopy. Rep. Prog. Phys. 66 523-582 (2003)

33. Wiesendanger, R. Spin mapping at the nanoscale and atomic scale. Rev. Mod. Phys. 81, 1495-1550 (2009).

34. Osterwalder, J. Spin-polarized photoemission. Lect. Notes. Phys. 697, 95-120 (2006).

35. Volnianska, O. \& Boguslawski, P. Magnetism of solids resulting from spin polarization of p orbitals. J. Phys. Condens. Matter 22, 073202 (2010).

36. Choi, W. H., Kang, P. G., Ryang, K. D. \& Yeom, H. W. Band-structure engineering of gold atomic wires on silicon by controlled doping. Phys. Rev. Lett. 100, 126801 (2008).

37. Mahan, G. D. Spin shift register from a one-dimensional atomic chain. Phys. Rev. Lett. 102, 016801 (2009).

38. Hellberg, C. S. \& Erwin, S. C. Strongly correlated electrons on a silicon surface: theory of a Mott insulator. Phys. Rev. Lett. 83, 1003-1006 (1999).

39. Otte, A. F. Can data be stored in a single magnetic atom? Europhys. News 39, 31-34 (2008)

40. Hirjibehedin, C. F. et al. Large magnetic anisotropy of a single atomic spin embedded in a surface molecular network. Science 317, 1199-1203 (2007).

41. Perdew, J. P., Burke, K. \& Ernzerhof, M. Generalized gradient approximation made simple. Phys. Rev. Lett. 77, 3865-3868 (1996).

42. Kresse, G. \& Hafner, J. Ab initio molecular dynamics for liquid metals. Phys. Rev. B 47, 558-561 (1993).

43. Kresse, G. \& Furthmüller, J. Efficient iterative schemes for ab initio total-energy calculations using a plane-wave basis set. Phys. Rev. B 54, 11169-11186 (1996).

44. Tersoff, J. \& Hamann, D. R. Theory of the scanning tunneling microscope. Phys. Rev. B 31, 805-813 (1985).

\section{Acknowledgments}

Helpful conversations with David L Huber are gratefully acknowledged. This work was supported by the Office of Naval Research, and by the NSF under award nos. DMR0705145 and DMR-0084402 (SRC). Computations were performed at the DoD Major Shared Resource Center at AFRL.

\section{Author contributions}

Both authors conceived and designed the research. S.C.E. performed the calculations and was the lead author of the article.

\section{Additional information}

Supplementary Information accompanies this paper on http://www.nature.com/ naturecommunications

Competing financial interests: The authors declare no competing financial interests.

Reprints and permission information is available online at http://npg.nature.com/ reprintsandpermissions/

How to cite this article: Erwin, S. C. et al. Intrinsic magnetism at silicon surfaces. Nat. Commun 1.58 doi: 10.1038/ncomms1056 (2010). 